\title{
Effect of bilateral and unilateral caloric vestibular stimulation in scopolamine induced dementia in Wistar albino rats.
}

\author{
Jinu KV ${ }^{1}$, Mukkadan JK ${ }^{1}$, Archana $\mathbf{R}^{2 *}$ \\ ${ }^{1}$ Department of Physiology, Little Flower Institute of Medical Sciences and Research, Angamaly, Kerala, India \\ ${ }^{2}$ Department of Physiology, Saveetha Medical College, Saveetha Institute of Medical and Technical Sciences \\ (SIMATS), Thandalam, Chennai, Tamil Nadu, India
}

\begin{abstract}
This study was undertaken to provide preliminary evidence for beneficial effects of both bilateral as well as unilateral caloric vestibular stimulation as an alternative therapy for enhancement of cognition and motor activity in Scopolamine induced dementia in Wistar albino rats. 36 healthy, adult male Wistar albino rats with body weight ranging from 150-200 $\mathrm{g}$ were used in the current study. Donepezil is used as standard drug in this study. Donepezil was administered orally at a dose of $5 \mathrm{mg} / \mathrm{kg}$ for 14 days. Caloric vestibular stimulation was administered by irrigating the middle ear cavity with hot water with a temperature of $\mathbf{4 5}$ degree centigrade with the help of syringe for $\mathbf{3 0}$ days. In conclusion, even though our study proves both unilateral left and bilateral hot water vestibular stimulation is beneficial in Scopolamine induced dementic rats in improving spatial cognition as well as motor control, unilateral left vestibular stimulation was found to be more beneficial. We recommend further detailed studies in this area to explore mechanisms of action.
\end{abstract}

Keywords: Caloric vestibular stimulation, Memory, Cognition, Dementia. Accepted on August 13, 2018

\section{Introduction}

We experience the surrounding world through our senses and our brain perceives and interprets the information from our senses, and makes us understand the world and our bodily orientation in it [1]. As the function and preciseness of brain start dementing due to various reasons, it compromises the quality of life in a larger extent. When a person develops dementia, brain's functionality is gradually reduced. People with dementia experiences recent memory loss (Asking same question repeatedly), difficulty in completing familiar task (Eg: cooking a meal), problems in communicating (Forgetting simple words or using wrong ones), disorientation (Getting lost on a familiar street), problems with abstract thinking (Eg: Dealing with money), misplacing things, mood changes, personality changes (depressed, anxious, irritable, suspicious, fearful) and incapable to focus on a certain task or to interact with the objects and people in the surrounding environment [2]. All these symptoms of dementia are progressive and the symptoms start out slowly and gradually get worse. So dementia is not a specific disease but a group of symptoms under one umbrella term. According to WHO, 47.5 million people have dementia worldwide and most common type of dementia is Alzheimer's disease and accounts for an estimated $60 \%$ to $80 \%$ of cases [3]. As there is no cure and no treatment that slows or stops its progression yet, an alarmingly growing dementia population makes the situation worse for the scientific community.

According to sensory integration theory, stimulating various senses affects the way we react to different situation. The history sensory stimulation especially vestibular stimulation researches dates back to late $18^{\text {th }}$ and $19^{\text {th }}$ century. Various techniques of vestibular stimulation were used to calm, soothe and even cure people for a range of psychiatric, neurological and neurodevelopmental conditions [4]. The continued research on vestibular stimulation has proven the beneficial effects in improving cognition and motor skills, relieving stress, improving depression and anxiety, promotes sleep, etc. Existing research describes clear links between symptoms of anxiety, depression and various aspects of cognition, particularly spatial memory and spatial perception, is associated with vestibular function [5]. All these beneficial effects are due to the massive network that connects vestibular nuclei to various brain areas like hippocampus, para-brachial nuclei, basal ganglia, cerebellum, thalamus and cerebral cortical areas which are collectively called vestibular cortex [6-13]. For dementia, drug treatment improve symptoms only temporarily, but not without side effects. So it's essential to have natural and healthy alternative therapies probably with a slight life style modification. Vestibular stimulation is a physiological approach for the management of cognitive and mood disorders. The emerging evidence proves that activation 
of the vestibular system, by experimental and natural methods is beneficial in the improving various aspects of memory as well as overall well-being [14]. This study was undertaken to provide preliminary evidence for beneficial effects of both bilateral as well as unilateral caloric vestibular stimulation as an alternative therapy for enhancement of cognition and motor activity in Scopolamine induced dementia in Wistar albino rats.

\section{Material and Methods}

\section{Animals}

A total of 36 healthy, adult male Wistar albino rats with body weight ranging between 150-200 $\mathrm{g}$ were used in the current study. Rats were housed in polypropylene cages $(30 \times 22 \times 14$ $\mathrm{cm})$, fed with standard-chow and water ad libitum. The animals were kept at a temperature of $22^{\circ} \mathrm{C} \pm 3^{\circ} \mathrm{C}$ and a $12 \mathrm{~h}$ light/dark cycle as well as a constant relative humidity throughout the experimental period. Rats are randomly assigned into six groups.

- G1: $(n=6)$ Negative control group

- G2: $(n=6)$ Positive control group -Scopolamine induced dementia

- G3: $(n=6)$ Scopolamine induced dementia+ Donepezil

- G4: $(n=6)$ Scopolamine induced dementia +bilateral hot water vestibular stimulation (30 days)

- G5: $(n=6)$ Scopolamine induced dementia +Unilateral Left ear hot water vestibular stimulation (30 days)

- G6: $(n=6)$ Scopolamine induced dementia+unilateral right ear hot water vestibular stimulation (30 days)

Scopolamine administration: $3 \mathrm{mg} / \mathrm{kg}$ Scopolamine was administered intraperitonially for 3 days to induce dementia in Wistar rats [15]. Scopolamine hydrobromide was purchased from Sigma-Aldrich (Bangalore, India).

Donepezil hydrochloride administration: Donepezil is used as standard drug in this study and administered orally at a dose of $5 \mathrm{mg} / \mathrm{kg}$ for 14 days [16]. Donepezil hydrochloride was purchased from Reddy's lab Hyderabad, India.

Caloric vestibular stimulation: Caloric vestibular stimulation was administered by irrigating the middle ear cavity with hot water with a temperature of $45^{\circ} \mathrm{C}$ with the help of syringe for 30 days [17]. In the present study, bilateral as well as unilateral vestibular stimulation (right and left ear individually) were used as a therapy.

\section{Behavioral tests}

Elevated plus maze: The elevated plus maze for rats consist of two open arms $(50 \mathrm{~cm} \times 10 \mathrm{~cm} \times 40 \mathrm{~cm})$ and two enclosed arms $(50 \mathrm{~cm} \times 10 \mathrm{~cm} \times 40 \mathrm{~cm})$ extended from a central platform $(5 \mathrm{~cm} \times 5 \mathrm{~cm})$ and the maze was elevated to a height of $58 \mathrm{~cm}$ from the floor. On the first day, each mouse was placed at the end of an open arm, facing away from the central platform. TL was defined as the time taken by the animal to move from the open arm into one of the covered arms with all its four legs. If the animal did not enter into one of the covered arm within $3 \mathrm{~min}$, it was gently pushed into one of the two covered arms and TL was assigned as $3 \mathrm{~min}$.

In our study EPM were used to measure Transfer latency (TL) which has been validated for cognitive behaviour [18]. Zero day values are recorded in all groups. Retention of this learnedtask (memory) was examined after Scopolamine induced standard drug treatment and 30 days of bilateral and unilateral vestibular stimulation.

Actophotometer: To rule out the beneficial effects of hot water vestibular stimulation on motor activity, horizontal locomotor activities of control and test animals were recorded for a period of $3 \mathrm{~min}$ using Medicraft actophotometer, model No: $600-40$, S. No: PA-0149, India. The units of the activity counts were arbitrary and based on the beam breaks by movement of the rats. Actophotometer test in rats "before and after administration of Scopolamine and 30 days of caloric (both bilateral and unilateral) vestibular stimulation" were used to validate motor coordination activity and anxiety level [19].

\section{Bio-chemical parameters}

Ach and AchE were measured by spectrophotometry. The animals were sacrificed after treatment by euthanasia. The whole brain was removed immediately and placed in ice-cold saline. The tissues weighing $0.5 \mathrm{~g}$ were homogenized with motor driven Teflon coated homogenizer with $5 \mathrm{~mL}$ of ice-cold $0.1 \mathrm{M}$ phosphate buffer $\mathrm{pH} 8.0$ to get $10 \%$ homogenate. The homogenate was centrifuged at $10,000 \mathrm{rpm}$ for $20 \mathrm{~min}$ at $5 \mathrm{C}$. The supernatant was collected and used for the ex vivo neurotransmitter estimation.

Estimation of brain acetylcholine esterase: The whole-brain AChE activity wasmeasured by spectrophotomety based on the method of Ellman et al. [20]. The principle behind assaying acetylcholine esterase activity was measured by following the increment of yellow colour produced from thiocholine when it reacts with dithiobisnitrobenzoate ion.

Procedure: $25 \mu \mathrm{L}$ of tissue homogenate was mixed with $75 \mu \mathrm{L}$ of phosphate buffer $(0.1 \mathrm{M} \mathrm{pH} \mathrm{8.0)}$ and $75 \mu \mathrm{L}$ of Dithiobisnitrobenzoic acid (DTNB) $(0.01 \mathrm{M})$ and the absorbance were measured at $415 \mathrm{~nm}$ after $5 \mathrm{~min}$ of incubation at room temperature. $25 \mu \mathrm{L}$ of substrate, Acetylthiocholine iodide (ATCI) was then added and the absorbance was measured again for $3 \mathrm{~min}$ at an interval of $1 \mathrm{~min}$. Change in absorbance per minute was thus determined.

Estimation of brain acetylcholine levels: Acetylcholine content in all the tissues was estimated by the method of Hestrin. The tissues were boiled to inactivate the enzyme and to release the bound Ach. Which reacts with ferric chloride, a brown colour developed was read at $540 \mathrm{~nm}$ against the reagent blank. The acetylcholine content was expressed as moles of acetylcholine/gram wet weight of tissue [21].

\section{Study setting}

The current study was conducted at Little Flower Medical Research Centre, Angamaly, Kerala, India. 


\section{Ethical consideration}

The experimental protocol was approved by Institutional Animals Ethics Committee (IAEC) of Little Flower Medical Research Centre. Animal care was taken as per the guidelines of CPCSEA.

\section{Data analysis}

Data was analysed by using SPSS 20.0 version. One-way analysis of variance was applied to observe the significance of difference between the groups. Bartlett's test for equal variances was applied for post hoc analysis. $\mathrm{P}$ value less than 0.05 was considered as significant.

\section{Results}

Figure 1 presents transfer latency on elevated plus maze before and after caloric vestibular stimulation in Scopolamine induced dementia in Wistar albino rats. Transfer latency was not significantly different between the groups before the treatment. There was a significant increase in the transfer latency in positive control group followed by administration of Scopolamine $(\mathrm{P}<0.001)$. Transfer latency was significantly decreased in Scopalamine+Donepezil group when compared with positive control group $(\mathrm{P}<0.001)$. However, when compared with the negative control group, the transfer latency in the Scopalamine+Donepezil group was significantly higher $(\mathrm{P}<0.001)$. Transfer latency was significantly decreased in Scopalamine+bilateral vestibular stimulation group when compared with positive control group $(\mathrm{P}<0.001)$. However, when compared with the negative control group, the transfer latency in the Scopalamine+bilateral vestibular stimulation group was significantly higher $(\mathrm{P}<0.001)$. Transfer latency was significantly higher in Scopalamine bilateral vestibular stimulation group when compared with Scopalamine + Donepezil group $(\mathrm{P}<0.01)$. Transfer latency was significantly decreased in Scopalamine+unilateral left vestibular stimulation group when compared with positive control group $(\mathrm{P}<0.001)$. However, when compared with the negative control group, the transfer latency in the Scopalamine+unilateral left vestibular stimulation group was significantly higher $(\mathrm{P}<0.001)$. Transfer latency was not significantly different between Scopalamine +unilateral left vestibular stimulation group and Scopalamine +Donepezil group. Transfer latency was not significantly different between Scopalamine+unilateral left vestibular stimulation group and Scopalamine+bilateral vestibular stimulation group. Transfer latency was not significantly different between Scopalamine+unilateral right vestibular stimulation group and positive control group. However, when compared with negative control group it was significantly higher $(\mathrm{P}<0.001)$. Transfer latency was significantly higher in Scopalamine+unilateral right vestibular stimulation group when compared with Scopalamine+Donepezil group $(\mathrm{P}<0.001)$. Transfer latency was not significantly different between Scopalamine+unilateral right vestibular stimulation group and Scopalamine+bilateral vestibular stimulation group. Transfer latency was significantly higher in Scopalamine +unilateral right vestibular stimulation group when compared with Scopalamine+unilateral left vestibular stimulation group $(\mathrm{P}<0.001)$.
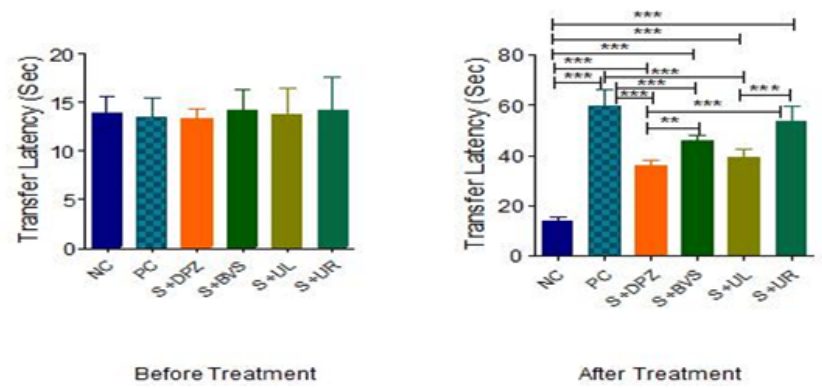

Figure 1: Transfer latency on elevated plus maze before and after treatment in Scopolamine induced dementia in Wistar albino rats. (Data was presented as Mean $\pm S D .{ }^{*} P<0.05$ is significant, ${ }^{* *} P<0.01$ is significant, ${ }^{* * *} P<0.001$ is significant). (NC: Negative Control; $P C$ : Positive Control; $S+D P Z$ : Scopalamine+Donepezil group; $S+B V S$ : Scopalamin+Bilateral Vestibular Stimulation group; $S+U L$ : Scopalamine+Unilateral Left Vestibular Stimulation group; $S+U R$ : Scopalamine+Unilateral Right Vestibular Stimulation group).

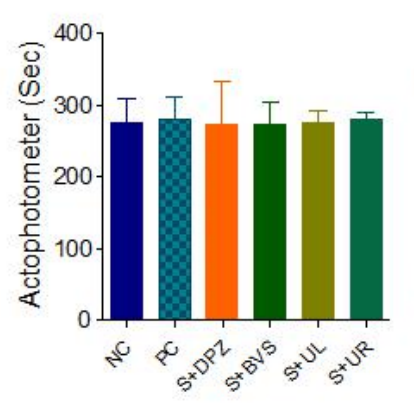

Before Treatment

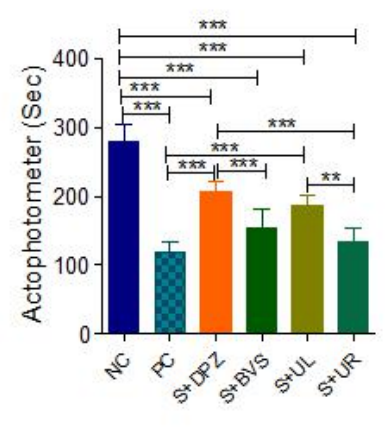

After Treatment
Figure 2. Actophotometer (sec) before and after treatment in Scopolamine induced dementia in Wistar albino rats (Data was presented as Mean $\pm S D .{ }^{*} P<0.05$ is significant, ${ }^{* *} P<0.01$ is significant, ${ }^{* * *} P<0.001$ is significant). (NC: Negative Control; $P C$ : Positive Control; S+DPZ: Scopalamine+Donepezil group; $S+B V S$ : Scopalamine+Bilateral Vestibular Stimulation group; $S+U L$ : Scopalamine+Unilateral Left Vestibular Stimulation group; $S+U R$. Scopalamine+Unilateral Right Vestibular Stimulation group).

Figure 2 explains motor co-ordination in Actophotometer before and after caloric vestibular stimulation. The scores are not significantly different in all groups before treatment. There was a significant decrease in movements in positive control after Scopolamine administration $(\mathrm{P}<0.001)$. The score has been significantly improved after the standard treatment when compared to positive control group $(\mathrm{P}<0.001)$. However, when compared with the negative control group, the score of Scopolamine+Donepezil is significantly lower $(\mathrm{P}<0.001)$. Score was not significant in Scopalamine+bilateral vestibular stimulation group when compared with positive control group but significantly lower with negative control and standard treatment group. Actophotometer score was significantly high 
in Scopalamine+unilateral left vestibular stimulation group when compared with positive control group $(\mathrm{P}<0.001)$. However when compared with negative control score is significantly lower $(\mathrm{P}<0.001)$. There is no significance of score among Scopolamine+Donepezil group and Scopolamine +unilateral left vestibular stimulation group. There is no significance between Scopolamine+unilateral left and Scopolamine+Bilateral vestibular stimulation group as well. The score was significantly lower in Scopalamine+unilateral right vestibular stimulation group when compared with Scopalamine+Donepezil group and negative control group $(\mathrm{P}<0.001)$. There was no significance between Scopalamine tunilateral right and positive control group. The score was significantly lower in Scopalamine+unilateral right vestibular stimulation group when compared with Scopalamine + unilateral left vestibular stimulationgroup $(\mathrm{P}<0.01)$.

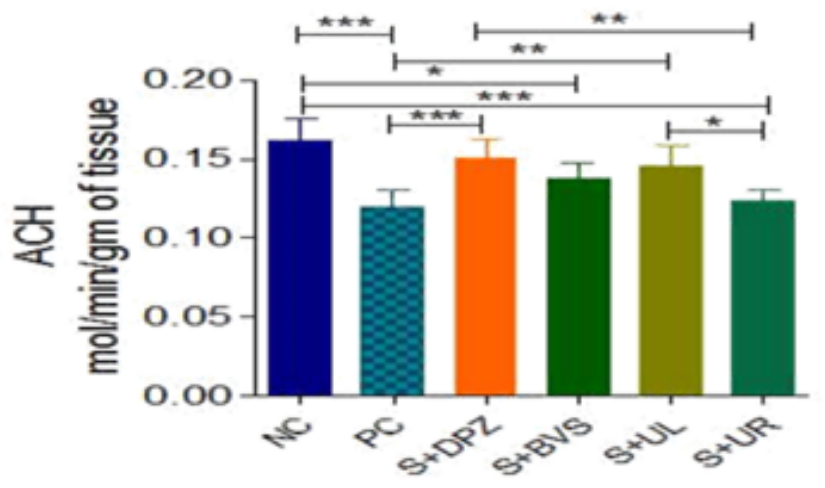

Figure 3. Acetyl choline levels in control and after treatment in Scopolamine induced dementia in Wistar albino rats. (Data was presented as Mean $\pm S D .{ }^{*} P<0.05$ is significant, ${ }^{* *} P<0.01$ is significant, ${ }^{* * *} P<0.001$ is significant). (NC: Negative Control; $P C$ : Positive Control; S+DPZ: Scopalamine+Donepezil group; S+BVS: Scopalamine+Bilateral Vestibular Stimulation group; $S+U L=$ Scopalamine+Unilateral Left Vestibular stimulation group; $S+U R$ : Scopalamine+Unilateral Right Vestibular Stimulation group).

Figure 3 explains Acetyl choline levels in control and treatment groups and it is expressed in $\mathrm{mol} / \mathrm{min} / \mathrm{g}$ of tissue. There was a significant decrease in Ach levels in positive control group followed by administration of Scopolamine $(\mathrm{P}<0.001)$. There was a significant increase in Ach level in Scopolamine+Donepezil group when compared with positive control. However, Ach levels were not significant between negative control and Scopolamine+Donepezil group. Ach levels in Scopolamine+bilateral vestibular stimulation group were significantly lower $(\mathrm{P}<0.05)$ when compared with negative control group. But there was no significance between positive control and Scopolamine+bilateral vestibular stimulation group. Ach levels were not significant between standard treatment group and Scopolamine+bilateral vestibular stimulation group as well. There was no significance in Ach levels among negative control, Scopolamine+Donepezil group, Scopolamine+bilateral vestibular stimulation groups and Scopolamine+unilateral left vestibular stimulation groups, but between positive control and unilateral left vestibular stimulation groups, Ach levels were significantly higher
$(\mathrm{P}<0.01)$ in Unilateral Left group. Ach levels were significantly lower in S+UR vestibular stimulation groups when compared to negative control $(\mathrm{P}<0.001)$. There was no significance between positive control and Scopolamine +unilateral right vestibular stimulation groups. But when compared to Standard treatment group and unilateral left vestibular stimulation group Ach levels were significantly lower $(\mathrm{P}<0.01$ and $\mathrm{P}<0.05)$. There wasn't any significance between bilateral and unilateral right vestibular stimulation groups.

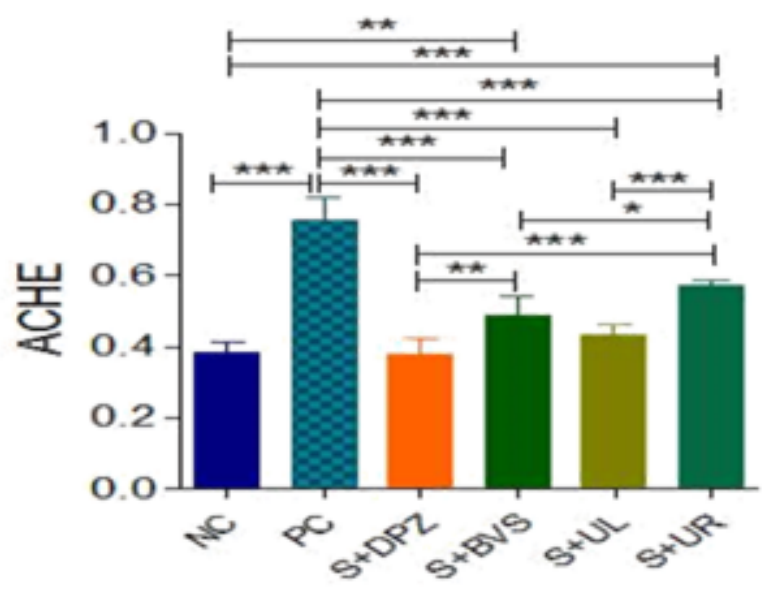

Figure 4. Acetyl choline esterase (AchE) activity in control and after treatment in Scopolamine induced dementia in Wistar albino rats (Data was presented as Mean $\pm S D .{ }^{*} P<0.05$ is significant, ${ }^{* *} P<0.01$ is significant, ${ }^{* * *} P<0.001$ is significant). (NC: Negative Control; $P C$ : Positive Control; S+DPZ: Scopalamine+Donepezil group; $S+B V S$ : Scopalamine+Bilateral Vestibular Stimulation group; $S+U L$ : Scopalamine+Unilateral Left Vestibular Stimulation group; $S+U R$ : Scopalamine+Unilateral Right Vestibular Stimulation group).

Figure 4 explains the AchE activity in Control and treatment groups. AchE activity significantly high in positive control group when compared to negative control $(\mathrm{P}<0.001)$. There was significance between negative control and Scopolamine + Donepezil groups. But AchE activity is significantly reduced in Scopolamine+Donepezil group when compared to positive control $(\mathrm{P}<0.001)$. In Scopolamine+bilateral vestibular stimulation group, AchE activity is significant when compared to negative control and Scopolamine+Donepezil groups $(\mathrm{P}<0.01)$. But when compared to positive control, AchE activity is significantly lower in Scopolamine+bilateral vestibular stimulation group $(\mathrm{P}<0.001)$. AchE activity levels were not significant among Scopolamine+unilateral left vestibular stimulation group, negative control and standard treatment groups. AchE activity were significantly lower in Scopolamine+unilateral left group when compared to PC $(\mathrm{P}<0.001)$. There wasn't any significance between Scopolamine+bilateral and Scopolamine+unilateral left vestibular stimulation groups. AchE activity is significantly increased in unilateral right vestibular stimulation group when compared to negative control and standard treatment groups $(\mathrm{P}<0.001)$. However compared with positive control, AchE activity is significantly lower in unilateral right groups $(\mathrm{P}<0.001)$. When compared with Scopolamine+bilateral and 
unilateral left groups, AchE is significantly higher in unilateral right group by $\mathrm{P}<0.05, \mathrm{P}<0.001$ respectively.

\section{Discussion}

In the present study we observed that caloric vestibular stimulation had improved spatial memory, motor co-ordination, increased Ach level and reduced AchE activity. It has been found that 30 days of unilateral left and bilateral vestibular stimulation have significantly improved transfer latency, actophotometer scores, Ach levels and decreased AchE activity compared to positive control. But unilateral right vestibular stimulation wasn't effective as unilateral left and bilateral vestibular stimulation. Unilateral left ear stimulation found more beneficial than bilateral as well as unilateral right ear vestibular stimulation in improving spatial memory and various neurotransmitter levels. Various studies have proven that bilateral vestibular loss leads to not only reflex deficits, but also attention and memory deficits, and anxiety disorders [22]. Long before itself vestibular stimulation were used to calm, soothe and even cure people with mental disorders. But unilateral stimulation studies are sparse. As the train of research on various types of vestibular stimulation and their beneficial effects continue, its therapeutic applicability on various neurodegenerative disorders is more and more revealing [23].

Vestibular stimulation influences cognitive process, anxiety and motor control through various complicated mechanisms. Vestibular system is extensively connected to the cognitive areas of the brain especially hippocampus, basal ganglia, parieto-frontal cortices, cerebellum which is collectively called vestibular cortices [24]. The theta rhythm generated in activating place cells and head direction cells in hippocampus by vestibular stimulation is important in cognitive functions like spatial orientation or spatial memory [25]. It has been proven that loss of vestibular function abolishes the theta rhythm generation [26], and could lead to bilateral hippocampal atrophy [27]. Acetylcholine is considered to be one of the important neurotransmitter involved in the regulation of cognitive functions. Vestibular stimulation is known to enhance various neurotransmitter level especially increase Ach level in CA1 area of hippocampus especially by activating septohippocampal cells there by Long Term Potentiation (LTP) [28]. Caloric vestibular stimulation does increase Ach release in rat hippocampus by $130 \%-160 \%$ than the basal level [29], and also inhibits AchE level in hippocampus [30]. So this natural therapy increases both the level and duration of action of Acetylcholine. The increment of dendritic arborization and synapses in the hippocampal pyramidal neurons by vestibular stimulation improves the performance in the spatial learning tasks [31]. Vestibular stimulation not only changes Ach levels but various neurotransmitters like serotonin, Glutamate, GABA, NorAdrenaline levels also changed by various types of vestibular stimulation. According to Samoudi et al. the increased GABA transmission in the ipsilesional substantia nigra followed by Stochastic Vestibular stimulation had an enhancing effect on locomotion in hemi-Parkinson rat [32]. Controlled vestibular stimulation decreasing the cortisol levels by inhibiting both the stress axis [33,34], thereby creates a stress-free condition, which also modulates learning and memory.

With all these past studies, bilateral vestibular stimulation is found beneficial in improving various aspects of brain function in neurodegenerative disorders. But only a few numbers of studies have done to explore the beneficial effects of unilateral vestibular stimulation (both right as well as left) in humans and animals [35]. In the present study we have used bilateral and unilateral right and left vestibular stimulation as well. Unilateral left vestibular stimulation has found beneficial in improving spatial memory and cognitive neurotransmitter levels over bilateral vestibular stimulation and it is probably due to asymmetrical stimulation of cerebral hemispheres [36].

Applying fMRI and PET methods in humans and animals, it turned out that the central processing of vestibular information is not symmetrical in two hemispheres. It depends on three determinants. It was demonstrated that subject's handedness, the side of the stimulated ear and the direction of the induced vestibular nystagmus [37]. It has been found that during caloric vestibular stimulation in right-handers right hemisphere is predominantly activated and in left-handers, left hemisphere activation is more with left ear stimulation. But various imaging studies and neuropsychological approaches have shown a stronger unilateral vestibular dominance in righthanders and a more bilateral representation in left-handers [38]. There is a stronger activation in the hemisphere ipsilateral to the irrigated ear. Activation is stronger in hemisphere ipsilateral to the slow phase of vestibular caloric nystagmus [39]. In human studies, it has been found that left caloric vestibular stimulation temporarily ameliorates left and right hemianesthesia [40], relieves the symptoms of visual hemineglect in right brain-damaged patient [41], and an immediate and sustained relief of symptoms of convulsion [42]. Lateralization and specialization of hemispheres in vestibular dominance is still unclear. Even though the vestibular processing structures were found bilaterally represented, which function is assigned to which hemisphere or one function itself choosing one hemisphere and therefore, displaces the other function onto the opposite hemisphere are highly unknown.

Over the last decades, it became clear that lateralization of cortical functions is not only an exclusive feature of the human brain but exists in animals also. Hemispheric dominance and lateralization of cortical functions is evident in many animal studies [43]. Best et al. provides primary evidence about the dominance of the left hemisphere in the processing of vestibular information independently of the "handedness" in rats. Trough Positron emission tomography images, it has been confirmed that left vestibular input was processed by more number of complex cortical and subcortical network, while right vestibular information was processed by fewer cortical areas. So left-sided vestibular information appears to be considered dominant compared to the right-sided information processing [44]. As per earlier study, vestibular information is processed by more cortical and sub-cortical clusters of neurons 
when left vestibular system is stimulating. There are lot other mechanism still need to uncover and explore. As evidences accumulation continued in vestibular research, it opened up limitless therapeutic opportunities even for most of the neurodegenerative disorders. The simplicity, lack of side effects, less time consumption and naturality makes it easy to incorporate in routine day-to-day life style not only to delay/ prevent neurodegenerative disorders, but also to improve the quality of life.

\section{Conclusion}

In conclusion, even though our study proves both unilateral left and bilateral hot water vestibular stimulation is beneficial in Scopolamine induced dementic rats in improving spatial cognition as well as motor control, unilateral left vestibular stimulation found more beneficial. We recommend further detailed studies in this area to explore mechanisms of action.

\section{Reference}

1. Fisher A, Murray E, Bundy A. Sensory integration theory and practice (2nd Edn). FA Davis Company, Philadelphia, 1991.

2. https://www.medicalnewstoday.com/articles/142214.php

3. http://www.who.int/mental_health/neurology/dementia/en

4. Grabherr L, Macauda G, Lenggenhager B. The moving history of vestibular stimulation as a therapeutic intervention. Multisens Res 2015; 28: 653-687.

5. Balaban CD. Projections from the parabrachial nucleus to the vestibular nuclei: potential substrates for autonomic and limbic influences on vestibular responses. Brain Res 2004; 996: 126-137.

6. Fiez JA. Cerebellar contributions to cognition. Neuron 1996; 16: 13-15.

7. Guldin WO, Grüsser OJ. Is there a vestibular cortex? Trends Neurosci 1998; 21: 254-259.

8. Brandt T, Schautzer F, Hamilton DA, Brüning R, Markowitsch HJ, Kalla R, Darlington C, Smith P, Strupp M. Vestibular loss causes hippocampal atrophy and impaired spatial memory in humans. Brain 2005; 128: 2732-2741.

9. Dominey J, Nighoghossian N, Denise P. Interaction between cortical control of vestibular function and spatial representation in man. Ventre Ann N Y Acad Sci 2005; 10: 494-497.

10. Smith PF, Geddes LH, Baek JH, Darlington CL, Zheng Y. Modulation of memory by vestibular lesions and galvanic vestibular stimulation. Front Neurol 2010; 1: 1-8.

11. Stackman RW, Taube JS. Firing properties of head direction cells in the rat anterior thalamic nucleus: dependence on vestibular input. J Neurosci 1997; 17: 4349-4358.

12. Shiroyama T, Kayahara T, Yasui Y, Nomura J, Nakano K. Projections of the vestibular nuclei to the thalamus in the rat: a Phaseolus vulgaris leucoagglutinin study. J Comp Neurol 1999; 407: 318-332.
13. Mizumori SJ, Puryear CB, Martig AK. Basal ganglia contributions to adaptive navigation. Behav Brain Res 2009; 199: 32-42.

14. Besnard S, Lopez C, Brandt T, Denis P, Smith PF. The vestibular system in cognitive and memory processes in mammalians. Front Integrat Neurosci 2015; 9: 1-4.

15. Rush DK. Reversal of scopolamine-induced amnesia of passive avoidance by pre-and post-training naloxone. Psychopharmacology 1986; 89: 296-300.

16. Sumanth M, Sowmya H, Nagaraj SV, Narasimharaju K. Efficacy of donepezil and galantamine in retrograde amnesia. Asian J Pharmaceut Clin Res 2010; 3: 23-25.

17. Horii A, Takeda N, Mochizuki T, Okakura-Mochizuki K, Yamamoto Y, Yamatodani A. Effects of vestibular stimulation on acetylcholine release from rat hippocampus: an in vivo microdialysis study. J Neurophysiol 1994; 72: 605-611.

18. Itoh J, Nabeshima T, Kameyama T. Utility of an elevated plus-maze for the evaluation of memory in mice: effects of nootropics, scopolamine and electroconvulsive shock. Psychopharmacology 1990; 101: 27-33.

19. DEWS PB. The measurement of the influence of drugs on voluntary activity in mice. Br J Pharmacol Chemother 1953; 8: 46-48.

20. Ellman GL, Courtney KD, Andres V, Featherstone RM. A new and rapid colorimetric determination of acetylcholinesterase activity. Biochem Pharmacol 1961; 7: $88-95$.

21. Hestrin S. The reaction of acetylcholine and other carboxylic acid derivatives with hydroxylamine, and its analytical application. J Biol Chem 1949; 180: 249-261.

22. Smith PF, Darlington CL, Zheng Y. Move it or lose it--is stimulation of the vestibular system necessary for normal spatial memory? Hippocampus 2010; 20: 36-43.

23. Grabherr L, Macauda G, Lenggenhager B. The moving history of vestibular stimulation as a therapeutic intervention. Multisens Res 2015; 28: 653-687.

24. Brandt $T$, Dieterich $M$. The vestibular cortex. Its locations, functions, and disorders. Ann N Y Acad Sci 1999; 871: 293-312.

25. Leutgeb S, Leutgeb JK, Moser MB, Moser EI. Place cells, spatial maps and the population code for memory. Curr Opin Neurobiol 2005; 15: 738-746.

26. Neo P, Carter D, Zheng, Y, Smith P, Darlington C, McNaughton N. Septal elicitation of hippocampal theta rhythm did not repair cognitive and emotional deficits resulting from vestibular lesions. Hippocampus 2012; 22: 1176-1187.

27. Stackman RW, Clark AS, Taube JS. Hippocampal spatial representations require vestibular input. Hippocampus 2002; 12: 91-303

28. Tai SK, Leung SL. Vestibular stimulation enhances hippocampal long-term potentiation via activation of cholinergic septohippocampal cells. Behav Brain Res 2012; 232: 174-182. 
29. Horii A, Takeda N, Mochizuki T, Okakura-Mochizuki K, Yamamoto Y, Yamatodani A. Effects of vestibular stimulation on acetylcholine release from rat hippocampus: an in vivo microdialysis study. J Neurophysiol 1994; 72: 605-611.

30. Devi NP, Mukkadan JK. Impact of rotatory vestibular stimulation in memory boosting. MOJ Anat\& Physiol 2017; 9: 144-151.

31. Devi NP, Mukkadan JK. Impact of rotatory vestibular stimulation and Myristica fragrans on spatial learning and memory in wistar albino rats. JIPBS 2016; 3: 17-32.

32. Samoudi G, Nissbrandt H, Dutia MB, Bergquist F. Noisy galvanic vestibular stimulation promotes GABA release in substansianigra and improves locomotion in hemiparkinsonian rats. Plos One 2012; 7: 1-10.

33. Kumar SS, Mukkadan JK. Can controlled vestibular stimulation reduce stress-A review. Health Sci 2013; 2: $1-13$.

34. Mody I, Maguire J. The reciprocal regulation of stress hormones and GABA(A) receptors. Front cell Neuro Sci. 2011; 6: 1-6.

35. Devi NP, JK Mukkadan. Effect of rotatory vestibular stimulation on learning and memoryin ratsstandardization of a novel method. Int J Pharm Pharm Sci 2017; 9: 145-151.

36. Bense S, Bartenstein P, Lutz S. Three determinants of vestibular hemispheric dominance during caloric stimulation: A positron emission tomography study. Ann N Y Acad Sci 2003; 1004: 440-445.

37. Dietrich M, Bense S. Dominance for vestibular cortical function in the non-dominant hemisphere. Cereb Cortex 2003; 13: 994-1007.

38. Schmidt L, Artinger F, Stumpf O, Kerkhoff G. Differential effects of galvanic vestibular stimulation on arm position sensein right vs. left-handers. Neuropsychologia 2013; 51: 893-899.

39. Moon SY. Caloric stimulation and optokinetic stimulation on Hemispatial neglect. J Clin Neurol 2006; 2: 12-28.

40. Bottini G, Paulesu M, Gandola MA. Left caloric vestibular stimulation ameliorates right hemianesthesia. Neurology 2005; 65: 1278-1283.

41. Rode G, Tilikete C, Luaute J, Rossetti Y, Vighetto A, Boisson D. Bilateral vestibular stimulation does not improve visual hemineglect. Neuropsychologia 2002; 40: 1104-1106.

42. Noll-Hussong M, Holzapfel S, Pokorny D. Caloric vestibular stimulation as a treatment for conversion disorder: A case report and medical hypothesis. Front Psychiatry 2014; 5: 1-8.

43. Corballis MC. Of mice and men - and lopsided birds. Cortex 2008; 44: 3-7.

44. Best C, Lange E, Buchholz HG. Left hemispheric dominance of vestibular processing indicates lateralization of cortical functions in rats. Brain Struct Funct 2014; 219: 2141-2158

\section{*Correspondence to}

Archana R

Department of Physiology

Saveetha Medical College

Saveetha Institute of Medical and Technical Sciences (SIMATS)

India 